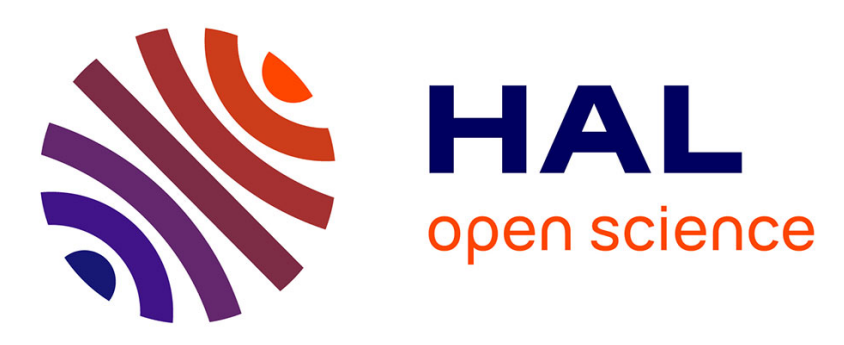

\title{
Monitoring of cracking and healing in an ultra high performance cementitious material using the time reversal technique
}

Sébastien Granger, Gilles Pijaudier-Cabot, Ahmed Loukili, David Marlot, Jean-Claude Lenain

\section{To cite this version:}

Sébastien Granger, Gilles Pijaudier-Cabot, Ahmed Loukili, David Marlot, Jean-Claude Lenain. Monitoring of cracking and healing in an ultra high performance cementitious material using the time reversal technique. Cement and Concrete Research, 2009, 39 (43), pp.296-302. 10.1016/j.cemconres.2009.01.004 . hal-00682156

\section{HAL Id: hal-00682156 https://hal.science/hal-00682156}

Submitted on 3 Jul 2017

HAL is a multi-disciplinary open access archive for the deposit and dissemination of scientific research documents, whether they are published or not. The documents may come from teaching and research institutions in France or abroad, or from public or private research centers.
L'archive ouverte pluridisciplinaire HAL, est destinée au dépôt et à la diffusion de documents scientifiques de niveau recherche, publiés ou non, émanant des établissements d'enseignement et de recherche français ou étrangers, des laboratoires publics ou privés. 


\title{
Monitoring of cracking and healing in an ultra high performance cementitious material using the time reversal technique
}

\author{
S. Granger ${ }^{\mathrm{a}}$, G. Pijaudier Cabot ${ }^{\mathrm{b}}$, A. Loukili ${ }^{\mathrm{a}}$, D. Marlot ${ }^{\mathrm{c}}$, J.C. Lenain ${ }^{\mathrm{c}}$ \\ ${ }^{a}$ REDO - Institut de Recherche en Génie Civil et Mécanique (GeM), UMR CNRS 6183, Ecole Centrale de Nantes, 1, rue de la Noë, F-44321 Nantes Cedex 3, France \\ ${ }^{b}$ Laboratoire des Fluides Complexes, Université de Pau et des Pays de l'Adour, ISA BTP, Allée du Parc Montaury, F-64600 Anglet, France \\ ${ }^{c}$ Euro Physical Acoustics, 27, rue Magellan, ZAC des Portes de Sucy, F-94370 Sucy en Brie, France
}

\begin{abstract}
A non-destructive testing technique based on the time reversal principle is applied to cementitious materials. Preliminary results are presented showing the reversibility of wave propagation and that focusing by time reversal is feasible in cementitious materials. Then, the technique is implemented on a mechanical test in order to monitor the cracking process of a concrete specimen loaded under three point bending. The acoustic mirror permits qualitative monitoring of the propagation of a crack by analyzing the focusing of a reference signal on a sensor placed near the expected crack path. A decrease of energy and of amplitude of the focused signal is observed upon cracking. Then, the specimen made of a material with high capabilities of self healing, is immersed in water, and healing processes are monitored. The results show a recovery of signal focusing upon healing. Comparisons with mechanical test data indicate that the method is sensitive to the variation of stiffness of the structure due to crack propagation and crack healing.
\end{abstract}

\author{
Keywords: \\ Acoustics \\ Time reversal \\ Cracking \\ Healing \\ Non-destructive technique
}

\section{Introduction}

Ultrasonic diagnostics is among the most widely used techniques for the detection of cracks in concrete structures. Historically, the propagation of ultrasonic pulses was considered first [1] and the determination of the pulse velocity is a standard in concrete testing [2]. Recent advances in ultrasonic testing rely on a more complete understanding of the dispersion of waves and scattering resulting into attenuation and diffusion [3], or on the analysis of backscattered waves [4]. In addition, ultrasonic techniques may complement other non-destructive testing methods such as electrical resistivity measurements, capacimetry or thermography (see e.g. Ref. [5]) in order to provide a better cartography of the current state of damage of a concrete structure. The purpose of this paper is to investigate the feasibility of another type of ultrasonic method based on the time reversal technique (described in detail by Fink $[6,7])$.

Time reversed acoustics is based on the invariance of the solution to the wave equation when time is reversed: in an elastic isotropic solid, without attenuation, the displacement field $\vec{u}$ resulting from wave propagation is the solution of the following equation:

$$
\begin{gathered}
\rho(\vec{r}) \frac{\partial^{2} \vec{u}(\vec{r}, t)}{\partial t^{2}}=(\lambda(\vec{r})+2 \mu(\vec{r})) \overrightarrow{\operatorname{grad}}(\operatorname{div}(\vec{u}(\vec{r}, t))) \\
-\mu(\vec{r}) \overrightarrow{\operatorname{rot}}(\overrightarrow{\operatorname{rot}}(\vec{u}(\vec{r}, t)))
\end{gathered}
$$

where $\vec{r}$ and $t$ are the space and time variables, $\lambda$ and $\mu$ are the Lamé coefficients, and $\rho$ is the mass density. Time derivatives of the second order appear only. Hence, the invariance of the solution to the wave equation, when time is reversed is verified as follows: if $\vec{u}(\vec{r}, t)$ is a solution of this equation, then $\vec{u}(\vec{r},-t)$ and by extension $\vec{u}(\vec{r}, T-t)$ (if the propagation is of limited duration $T$, which is often the case in practice) are also solutions of this equation. If losses of energy are weak in the considered material, signals emitted from a source and detected, can be re-emitted to their source after having been time reversed. As in classical optics, this reversibility ensures that the waves converge in a synchronized way to the original source, re-creating the initial signal. In theory, the proper re-creation of the initial signal relies on elastic properties. However, material heterogeneity will result into a more complex wave propagation process, with multiple reflections and refraction. Still, if the linearity of the wave propagation process is preserved, the heterogeneities of the material are without importance on the reversibility. Therefore the method fits very well to strongly 
Time reversal mirror

(on the other face of the specimen)

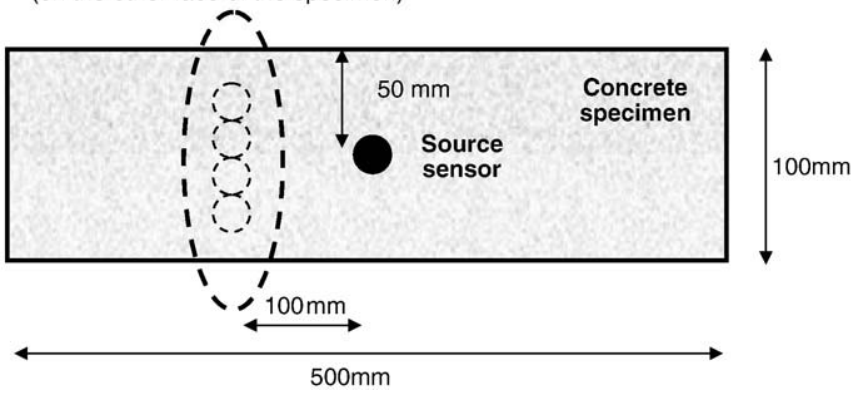

Fig. 1. Preliminary tests configuration - position of the source sensor and of the mirror.

heterogeneous materials such as concrete. The limitation of the time reversal principle is the possible non linear effects such as attenuation which may also be influenced by wave dispersion. Taking into account the inevitable dissipation and attenuation, the initial signal is not completely recovered. The convergence to the original source location, also known as focusing, and the recomposition of the original signal are not perfect. The focused signal is then characterized, for instance, by the maxima in amplitude and energy and by its frequency spectrum.

In theory, the signal has to be detected in all directions from the source (over the entire surface of the specimen) in order to be optimally re-created after time reversal. In practice, this is not feasible, and the signal after emission from the source is detected only over a limited area of the boundary of the specimen, called a time reversal mirror. The mirror consists in a network of transducers and electronic devices capable of recording signals, and re-emitting them in the opposite direction. This appealing possibility of detecting signals on a limited area of the specimen, and still being able to focus on the initial source location upon re-emission, is related to works carried out on reverberating cavities $[7,9]$. The time reversal technique does not take into account only the signals propagating directly from the source to the detectors (and in the opposite direction). Detection on sensors networks may also include, in addition to direct waves, the waves coming from all the reflections that can occur for the signal generated by the source. These additional waves are also recorded at the surface of the specimen: upon time reversal they improve the focusing drastically. The more reflected waves the recorded signal contains, the better the resolution during focusing [8]. As a consequence of the multiple reflections, the acoustic waves detected are signals with a random distribution of amplitude, called codas. Draeger and Fink [9] have observed such codas in silicon wafers, and they have shown that time reversal is possible in such specimens using only one source sensor and one mirror sensor, due to the improvement of the temporal information of the signal by the multiple reflections. The time reversal procedure can also be iterated. It provides, in the case of multiple sources or targets, a selective focusing on the most reflective source. A method, called DORT (French acronym for decomposition of the time reversal operator), has been developed in order to do this numerically [10].

The variety of applications of the time reversal technique is large. Among the most common applications, we can quote the detection of defects in materials, possibly coupled with imaging techniques [11-13]. Others applications are being developed in the medical field for imaging, tumour detection, and destruction of stones in kidneys [14-16]. Further developments deal for instance with underwater communication techniques [7]. The time reversal principle has never been used for the detection of cracks in concrete or cementitious specimens and this is the aim of this paper. Section 2 presents the principle of the non-destructive investigation technique and the instrumentation. Section 3 reports preliminary focusing tests aimed at investigating its sensitivity on a specific material: an ultra high performance cementitious material. Section 4 discusses the detection of cracking and healing in this material.

\section{Principle and instrumentation}

One aspect of time reversal is to be able to propagate waves in the reverse direction and to synchronize them on the original source location in order to reconstruct the original signal. This focusing is influenced by the changes which can occur in the specimen between the time of the initial emission of the signals and the time of reemission after time reversal. The variation of focusing is an indicator of the presence of defects in the monitored specimen.

The methodology consists in three phases. The first one is the emission in the specimen of a controlled wave. The location where the wave is emitted is in the region where cracking and damage are expected. It is at this location that focusing will be analyzed later on. The corresponding signal is recorded by the acoustic mirror. The signal content is the incident wave and the multiple reflected waves from the boundaries (and heterogeneities) of the specimen. This signal is timereversed in order to be re-emitted. If the material properties in the specimen do not change, upon re-emission of the signal, the same focusing process will occur each time at the location where the signal was emitted initially. If the material properties evolve, a variation of the focused signal is expected and it is this drift of the focused signal which will serve as an indicator of damage. Note that there may be several regions monitored simultaneously in a given structure; the a

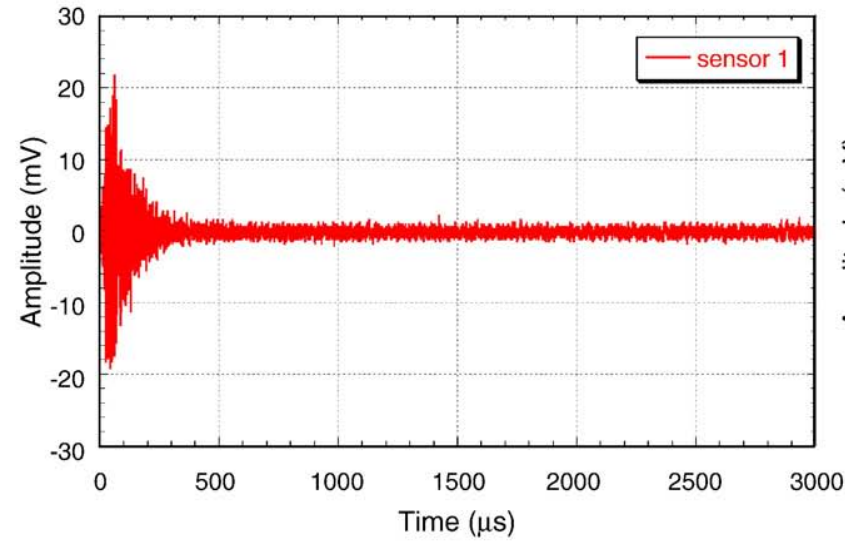

b

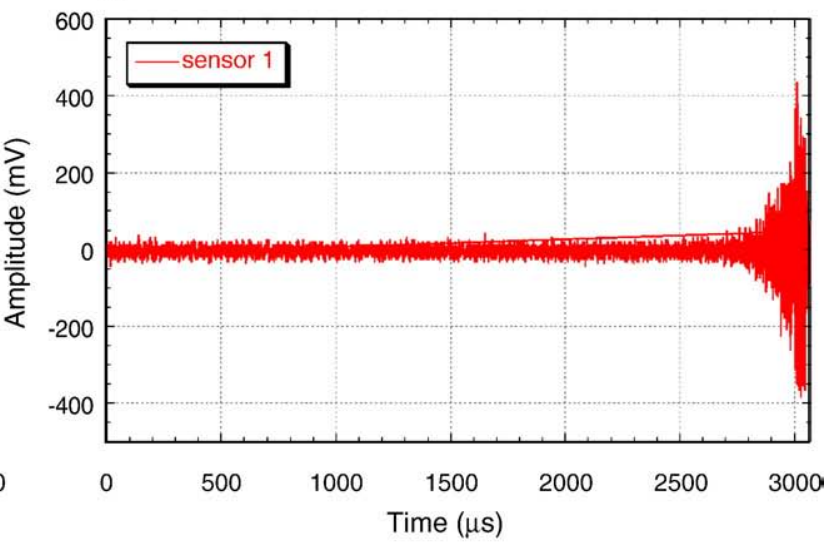

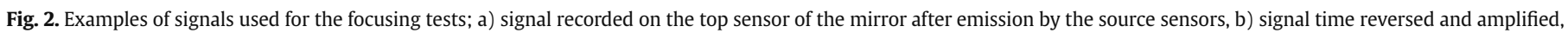
reemitted by the top sensor of the mirror. 
procedure is repeated for each zone where damage is monitored. There are several locations from which the wave is emitted and where the signal recorded by the acoustic mirror is focused. One may use several acoustic mirrors, each one placed nearby a monitored location or a single acoustic mirror if the wave attenuation is not important.

An important issue in the development of this non-destructive technique is the specification of transducers. Generally, for time reversal applications, transducers used are ultrasound ones. The same sensors are used both for emission and recording and good capabilities are required for the sensors in order to do that. The sensors used for this study are piezoelectric with a diameter of $10 \mathrm{~mm}$ (type SOMAXIS). They have a resonance frequency at $1 \mathrm{MHz}$, but they are broadband with a high sensibility between 0 and $50 \mathrm{MHz}$. These sensors have been used for pulse generation, for periodic recording and for the acoustic mirrors.

The acoustic mirror comprises 4 sensors and a time reversal system with 4 channels able to record, to reverse and to send signals. The signals are acquired with a system designed for acoustic emission applications [17]. It allows recording of arbitrary signals (whatever the duration, amplitude, energy...) digitized with a chosen sampling rate. The signals are recorded in a synchronized way, in the sense that recording begins for all the transducers when a single sensor is excited. Moreover, the system permits the extraction of useful signal characteristics (amplitude, energy, duration...). The incoming signals, after being recorded by the four transducers are digitized, time-reversed (first in-last out), amplified if needed, and stored. Then, they are ready to be re-emitted by the sensors. For the re-emission, the system comprises waveforms generation cards which are capable of emitting any kind of signal. The four generation cards are synchronized to an external trigger (a pulse generator in this case), and the four signals are send simultaneously by the four sensors of the mirror.

\section{Focusing tests in an ultra high performance cementitious material}

The first step was to carry out a very simple focusing test in order to ensure the feasibility of the method. These tests have been performed on an ultra high performance cementitious material (UHPC) distributed by Lafarge called "Ductal". It is characterized by a low water to cement ratio ( $w / c$ ratio), close to 0.2 . The composition of this UHPC includes cement, silica fume, sand (maximum aggregate size $2 \mathrm{~mm}$ ), water and superplasticizer, but no coarse gravel. This is the same material as in the experimental programme reported by Granger et al. [17]. We have a quite homogeneous material, with an attenuation lower than that of ordinary concrete. These characteristics have been chosen for the sake of simplicity, in order to avoid some potential

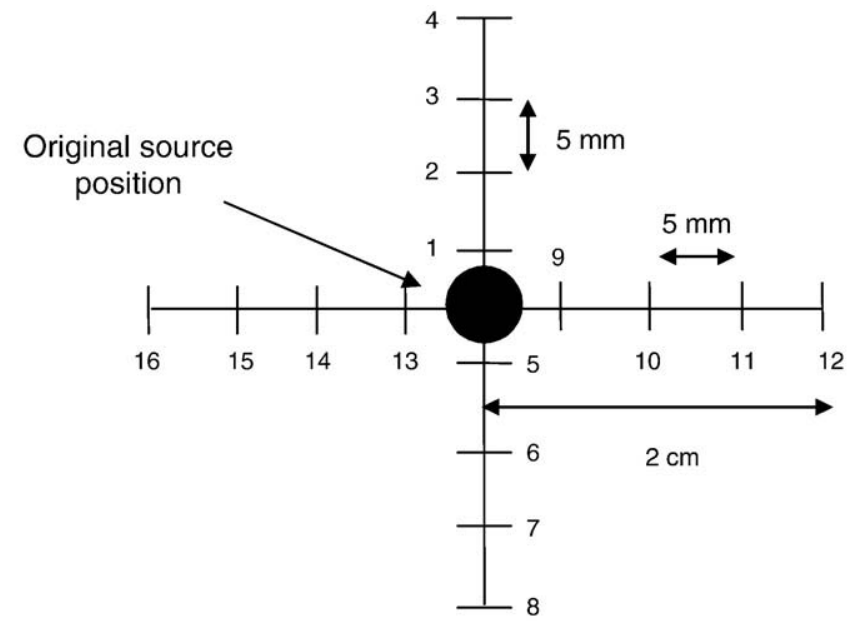

Fig. 3. Numbering of the different positions of focusing analysis from the original source position (related to Fig. 1).

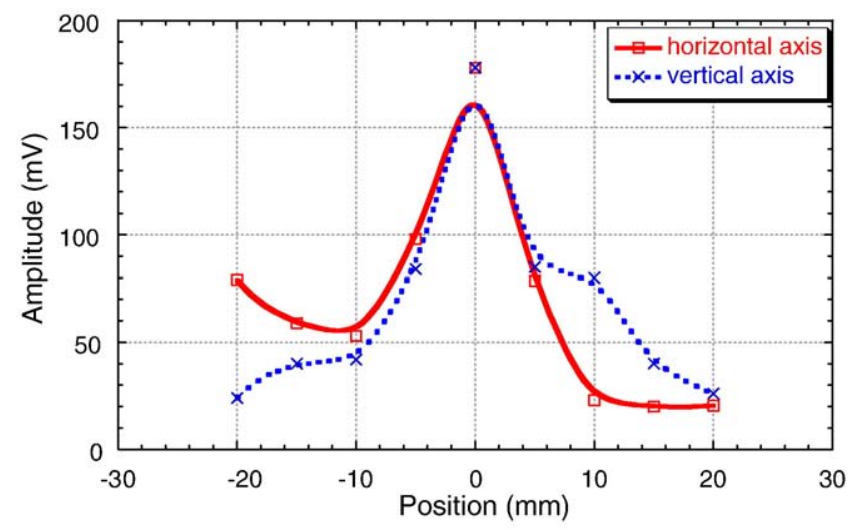

Fig. 4. Amplitude of the signal detected after time reversal and reemission, as a function of the position with respect to the source location (related to Fig. 3).

complications. Attenuation should degrade the focusing of the signal with less energy and a decrease of amplitude. Since our purpose is to record the drifting with time in the focusing of the signal, this may not be very important, but still it may result into a loss of accuracy, with smaller relative variations of amplitude. Application to ordinary concrete will be the subject of further studies, after tests on UHPC which is almost an ideal material have proven the efficiency of the method. Since the technique relies on the reversibility of acoustic waves, the heterogeneity should not be a major difficulty when applying it to a material that contains larger aggregates in a more porous matrix. Attenuation should be the main issue that will arise for ordinary concretes. Its influence on the quality of focusing and on the subsequent drifts of focusing due to material damage remains to be evaluated (also in the case of reinforced concrete where a degradation of the steel-concrete bond may have an influence too).

The focusing tests have been performed on prismatic specimens, with dimensions of $50 \times 100 \times 500 \mathrm{~mm}^{3}$. The principle of these tests is based on the work carried out by Draeger and Fink [9] on reverberating cavities. As can be seen in Fig. 1, the UHPC specimen was instrumented with 5 sensors, able to send and record waves, which are coupled to the specimen with a silicone glue. A single sensor was placed on one face of the specimen and acts as the original wave source. The four others are placed together on the opposite face and represent the acoustic mirror.

The first step consists in emitting a signal from the source sensor, and then recording the signals detected on the time reversal mirror. These detected signals are time-reversed and then amplified. The signal emitted by the original source is a pulse of duration $0.5 \mu \mathrm{s}$. The

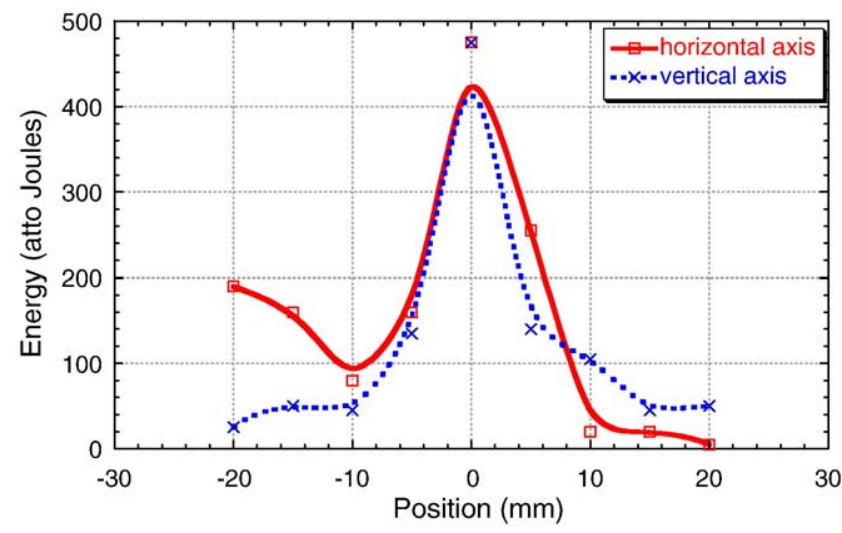

Fig. 5. Energy of the signal detected after time reversal and reemission, as a function of the position with respect to the source location (related to Fig. 3). 


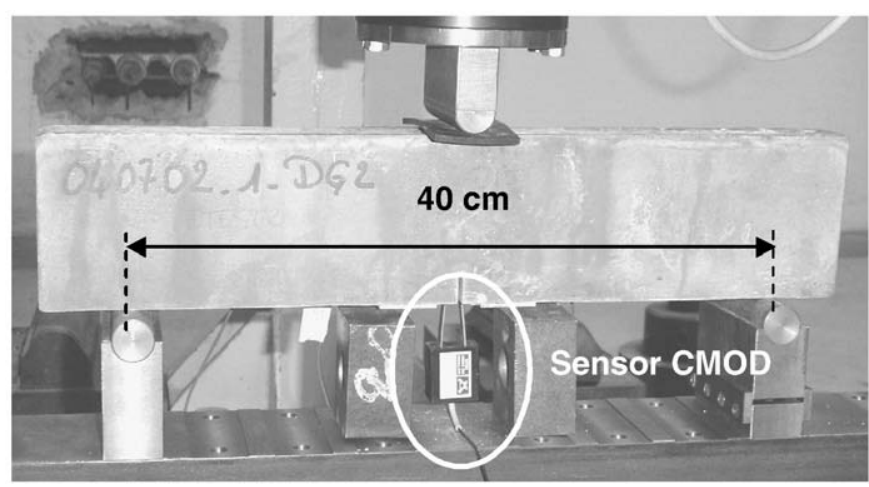

Fig. 6. Three points bending test configuration.

detected signals are recorded on the mirror in a synchronized way, with a sequence of 15,000 points and a frequency of $5 \mathrm{MHz}$ for each signal, which corresponds to durations of $3000 \mu \mathrm{s}$. Amplification is performed in order to overcome some attenuation processes (due to the sensors and their coupling to the specimen especially) during the reemission of the signals in the second step of the experimental procedure. The four time-reversed signals are amplified in the same way, so as to keep their same relative value. Here, the amplitudes of each signal are multiplied by 20 , in order to reach the maximum value that can be sent by the signal generation cards. An example of the signal detected on one sensor of the mirror is presented in Fig. 2, along with the corresponding time reversed and amplified signal. The final step of the tests is then the reemission of the time reversed signals in the specimen, in a synchronized way. The focusing of the signal is then analyzed at the original source location and nearby with a sensor that is moved in order to scan the surface of the specimen.

Fig. 3 represents the positions where focusing is analyzed horizontally and vertically from the original source. Fig. 4 displays the amplitude of the signal which has been detected after time reversal and re-emission, as a function of the position of detection. The maximum of amplitude detected is for the original position of the sensor where the focusing is optimum. The amplitude is divided by more than 1.5 at a distance of $5 \mathrm{~mm}$ from the original source, and by more than 3 at $15 \mathrm{~mm}$ from the source. Fig. 5 presents the energy of the focused signal as a function of the distance from the source. Ideally, if the size of the sensors would be negligible, the signal should focus at the source location only and no signal should be recorded elsewhere. Here, some signal can be recorded nearby the source location but the amplitude and energy are small. Considering that the diameters of the sensors are $10 \mathrm{~mm}$, we may conclude that the focusing is narrow, consistent with the instrumentation capabilities. Upon a change of material properties near the source location, the focusing is expected to degrade, i.e. reduce signal amplitude and energy. Note that it is the size of the focusing zone that may increase in tests on ordinary concrete, due to attenuation and wave dispersion. This, in turn, should yield a smaller variation of the amplitude and energy, and difficulties in the detection of relative variations with time if they are too small.

\section{Monitoring of cracking and healing in an ultra high performance cementitious material}

The UHPC used for this work is characterized by a very low $\mathrm{w} / \mathrm{c}$ ratio. This implies a large amount of unhydrated cement in the hardened microstructure (50 to 60\% [18]) which can contribute to self healing of potential cracks [19-21]. A previous study performed on this UHPC has shown that cracks can heal when specimens are totally immersed in water [17], even in the first days and weeks of immersion. We have used the time reversal method to monitor the cracking process and the potential healing.

Prismatic specimens with dimensions of $50 \times 100 \times 500 \mathrm{~mm}^{3}$ have been used. The first step of the test is the crack initiation and propagation, and its monitoring. The concrete specimen is loaded under three points bending. A notch of depth $20 \mathrm{~mm}$ and thickness $1.5 \mathrm{~mm}$ is pre-formed in the specimen by placing a PMMA plate in the mould. The mechanical test is crack mouth opening displacement (CMOD) controlled with a constant rate of $0.05 \mu \mathrm{m} / \mathrm{s}$. The CMOD sensor is placed between two steel plates stuck on each side of the notch on the bottom face of the specimen (see Fig. 6).

The objective is to achieve controlled cracking of the specimen and to evaluate the consequences of crack propagation on the focusing of a signal generated before cracking. For the time reversal method, the principle depicted in the previous section is kept. The acoustic set-up is similar to the one used for the previous focusing tests. The source sensor is placed on the expected crack path due to the notch. It could have been placed nearby, with the same result since the crack tip does not reach the sensor during the test. During the propagation of the crack, the focusing of the acoustic signal on this location should be affected by the crack propagation and the diffuse damage surrounding the crack. The test set-up is depicted in Fig. 7.

\subsection{Monitoring during cracking}

The first step consists in emitting a signal with the sensor placed on the expected crack path, before the mechanical load is applied. The signals detected by the mirror are then time reversed, amplified and (a)

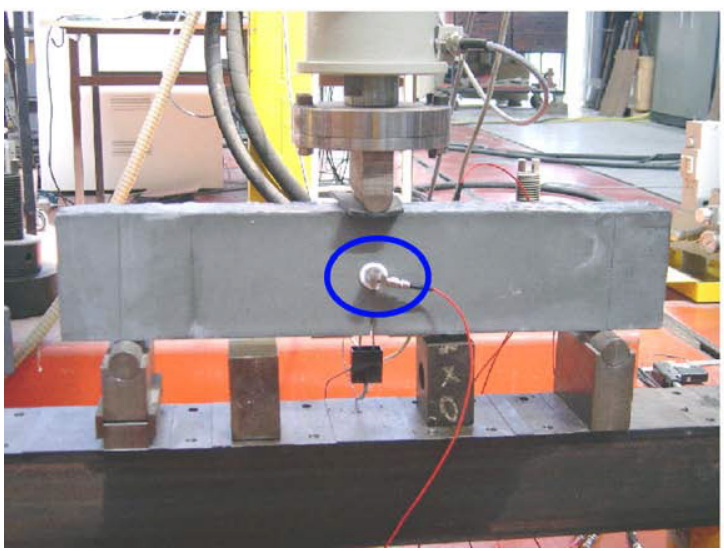

(b)

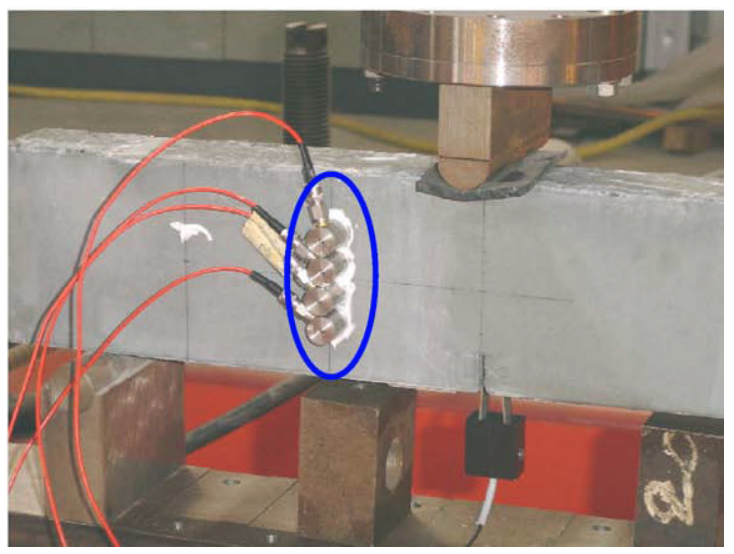

Fig. 7. Time reversal instrumentation during the mechanical test; a) sensor nearby the expected crack path, b) time reversal mirror on the other face of the specimen. 


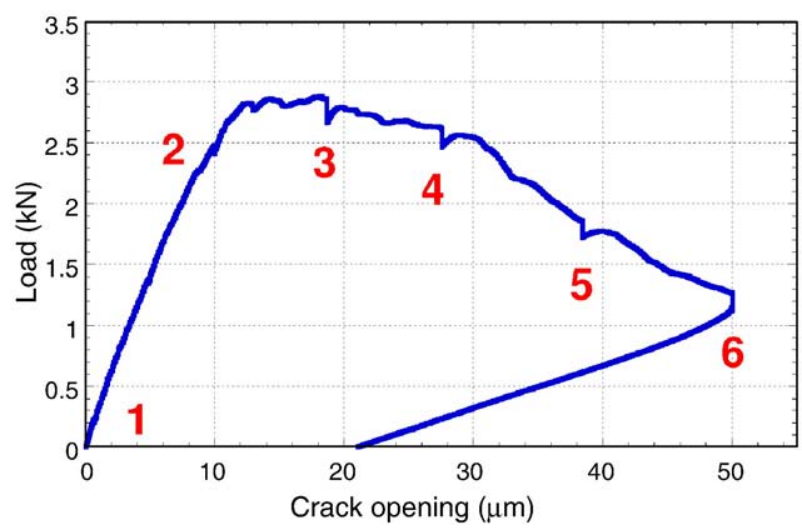

Fig. 8. Mechanical test result and the different steps of focusing tests during the cracking process.
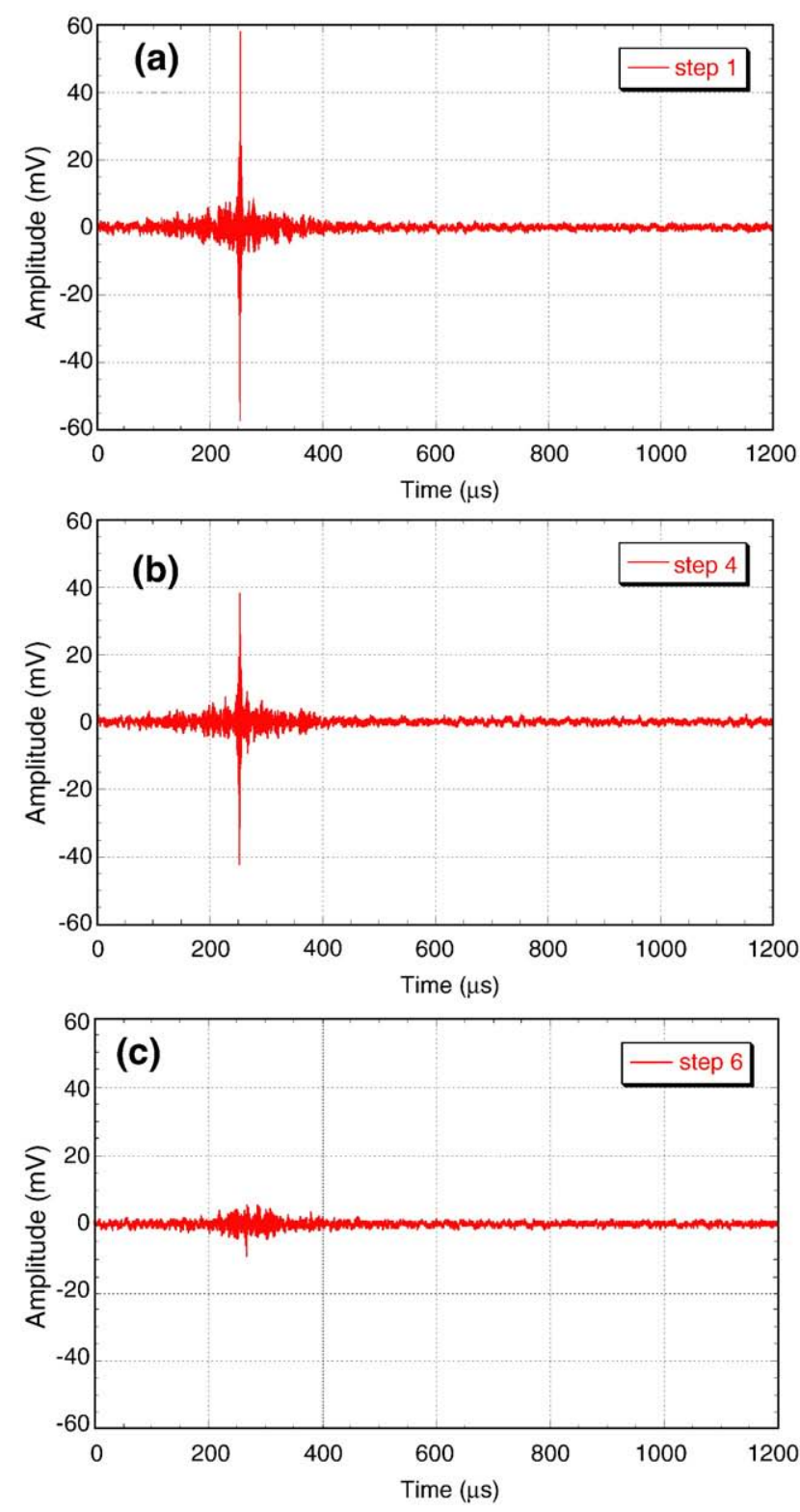

Fig. 9. Signal recorded on the source sensor after time reversal and reemission, at steps 1, 4, and 6 (see Fig. 8).

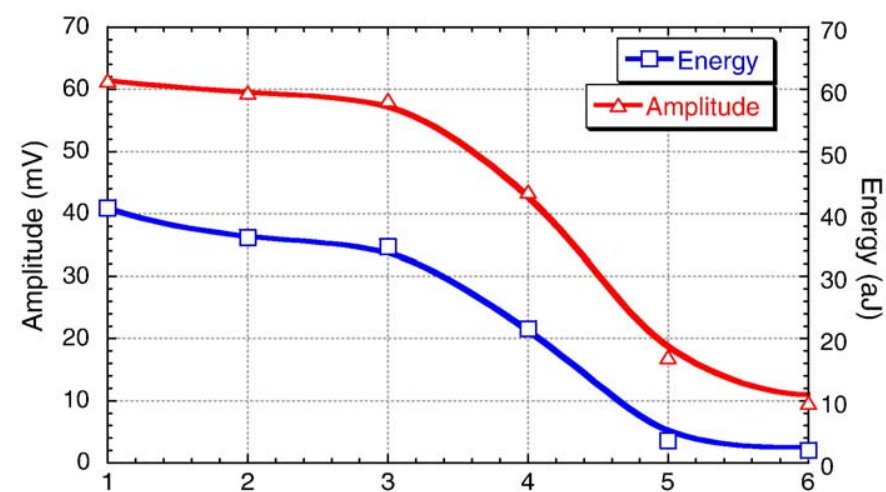

Fig. 10. Evolution of the energy and amplitude of the focusing signals detected at the different states after time reversal during the cracking process (see Fig. 8).

stored for further re-emission in the specimen as in the initial focusing tests (see Section 3). The signal detected at the source location by the sensor after re-emission, without damage, is kept as the reference. During the mechanical tests, the stored signals are re-emitted periodically, at different states of crack propagation. The signal (amplitude, energy and frequency spectrum) at the source location is recorded, analyzed and compared to the reference. It is the recomposition at the source location of the signal recorded initially by the acoustic mirror that is compared to the reference. Note that during cracking and healing, the five sensors stay coupled to the specimen in order to avoid bias due to a possible imperfect coupling between the sensors and the specimen.

Fig. 8 presents the result of the mechanical test associated with the experiment. The curve displays the crack opening versus the applied load. Cracking is performed in the post peak regime. The residual crack opening after unloading (which is also crack opening controlled, with the same absolute rate as loading) is close to $20 \mu \mathrm{m}$. On this figure, the different steps where time reversal monitoring is performed are denoted as 1 to 6 . During the monitoring (typically $5 \mathrm{~min}$ ), the mechanical test is temporarily stopped to a constant crack opening and a very slight relaxation occurs. Fig. 9 shows signals that have been recorded on the source location after time reversal, at different cracking steps. The first one, in Fig. 9a, is the reference one, while the specimen is undamaged. The two others (Fig. 9b,c) correspond to damaged states, respectively points 4 and 6 in Fig. 8. The evolution of the signal with the propagation of the crack is already significant. Initially, we observe an

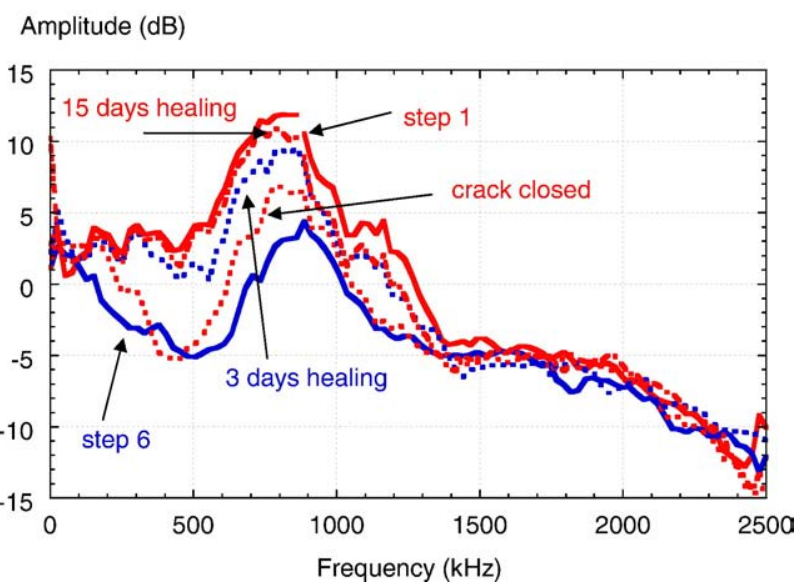

Fig. 11. Frequency spectrum of the focusing signals detected at the different states afte time reversal during the healing process. 


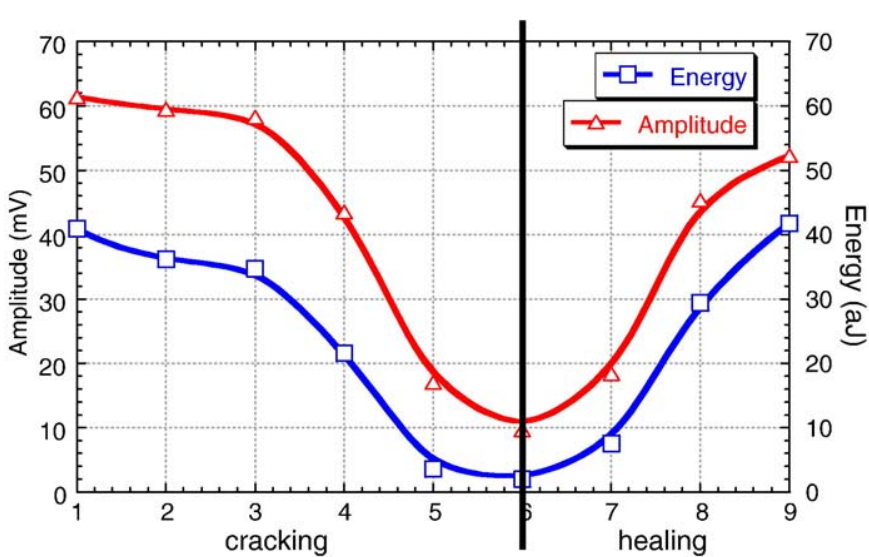

Fig. 12. Evolution of the energy and amplitude of the focusing signals detected at the different states after time reversal during the cracking and healing processes.

amplitude close to $60 \mathrm{mV}$, but by step 6 this amplitude has been divided by more than 6 . A more illustrative analysis is provided on the evolution of the energy and of the amplitude of the signal (Fig. 10). On this figure, the $x$-axis represents the different steps, as mentioned in Fig. 8. So we have a very significant decrease of the amplitude and of energy of the signal upon crack propagation.

\subsection{Monitoring during healing}

After this cracking phase, and according to the methodology presented in Ref. [17], the specimen is totally immersed in tap water in order for the crack to heal. Sensors are protected during immersion. After 3 days and 15 days of healing, monitoring of the self healing was performed.

Fig. 11 shows the evolution of the frequency spectrum of the signal detected at the source location at the different states: steps 1 and 6 of Fig. 6 (i.e. before cracking, and at the end of cracking), just after the unloading of the concrete specimen (crack closing) and after 3 and 15 days of immersion in water. The reference spectrum is also represented in the figure. The peaks on this figure corresponds to the resonance frequency of the transducers. It is not the shape of the spectrum which is of interest but rather its evolution with damage and self healing. There is a decrease of amplitude upon microcracking and then an increase of amplitude with the time of healing. Variations are quite noticeable in the low frequency regime, below the resonance frequency of the transducers. Fig. 12 presents the variations of energy and of amplitude of the focused signal detected at the source location. The cracking part is also reproduced, before healing. in this figure, step 7 corresponds to unloading and steps 8 and 9 correspond to 3 days and 15 days of healing respectively. After the decrease during cracking, it can be clearly seen that the characteristics of the focused signal increase again, suggesting crack healing. Self healing of the microstructure inside the crack for similar specimens was already observed in Ref. [17], on mechanical and acoustic emission analyses. Cracking reduced the beam stiffness to $14 \%$ of the initial one, and according to the experiments presented in Ref. [17], after 3 days of healing the bending stiffness should reach $38 \%$ of the initial bending stiffness, and after 15 days, it should reach $75 \%$ of the initial stiffness. The present method relies on wave propagation and reversibility. Therefore it is expected to be sensitive to the stiffness of the structure, but not necessarily to its strength. As explained in Ref. [17], the strength was not increasing with the same rate. This is probably due to the fact that hydration occurs between the two crack faces, which is a different environment compared to the original hydration, with a different resulting microstructure. The time reversal technique or acoustic emission techniques cannot resolve the difference between the original microstructure and the microstructure of the same material after healing.

\section{Conclusion}

A novel non-destructive technique based on time reversal of acoustic waves has been investigated for the indirect monitoring of cracking and damage. It is based on the focusing of a signal emitted initially in the structure, whose drift can be considered as a damage indicator. It has been applied to an ultra high performance concrete, which has the particularity to have a low attenuation, and to have capacities of self healing.

Preliminary tests have been conducted in order to exhibit the focusing of the signal upon time reversal in uncracked specimens. The results show that a signal can be resent to its source after having been recorded, time reversed, amplified and re-emitted in the material by a time reversal mirror. The accuracy of the focusing is satisfactory considering the size of the sensors.

A prismatic bending specimen has been instrumented with the developed device, and cracking and healing were analyzed. In order to do that, a sensor was placed near the expected crack path, defined by the notch of the specimen submitted to bending. The signals at this sensor, after time reversal, have been analyzed at different stages of cracking and healing. The amplitude of the signal and its energy decrease upon cracking and increase again upon crack healing. Comparisons with mechanical test data show that the method is sensitive to the variation of stiffness of the structure due to crack propagation and crack healing.

Promising results have been obtained but it is clear that the drift of the focused signal as a function of material damage needs some proper quantitative analysis and a detailed understanding. After that, extensions to ordinary concrete and to reinforced concrete structure might be considered. Attenuation should be the main difficulty that may arise for ordinary concretes. In the case of reinforced concrete, the degradation of the steel-concrete bond is an additional mechanism and the capability of the time reversal technique to characterize damage on this interface needs to be investigated.

\section{Acknowledgements}

Financial supports from the "Région des Pays de la Loire" and from the partnership between Lafarge and the R\&DO group are gratefully acknowledged.

\section{References}

[1] R. Jones, The non destructive testing of concrete, Magazine of Concrete Research 2 (1949) 67-78.

[2] K. Komlos, S. Popovics, T. Nurnbergerova, B. Babal, J.S. Popovics, Ultrasonic pulse velocity test of concrete properties specified in various standards, Cement \& Concrete Composites 18 (1996) 357-364.

[3] P. Anugonda, J.S. Whien, J.A. Turner, Diffusion of ultrasounds in concrete, Ultrasonics 39 (2001) 429-435.

[4] J.F. Chaix, V. Garnier, G. Corneloup, Concrete damage evolution analysis by backscattered ultrasonic waves, NDT \& E International 36 (2003) 461-469.

[5] D. Breysse, G. Klysz, X. Dérobert, C. Sireix, J.F. Lataste, How to combine several nondestructive techniques for a better assessment of concrete structures, Cement and Concrete Research 38 (2008) 783-793.

[6] M. Fink, Time reversal of ultrasonic fields - Part 1: basic principles, IEEE Transactions on Ultrasonics, Ferroelectrics, and Frequency Control 39 (5) (September 1992).

[7] M. Fink, Time reversed acoustics, Scientific American (November 1999) 67-73.

[8] N. Quieffin, "Etude du rayonnement acoustique de structures solides: vers un système d'imagerie haute résolution", pHD Thesis, University of Paris 6, 2004.

[9] C. Draeger, M. Fink, One channel time reversal of elastic waves in a 2D silicon cavity, Physical Review Letters 79 (1997) 407-410.

[10] C. Prada, E. Kerbrat, D. Cassereau, M. Fink, Time reversal techniques in ultrasonic nondestructive testing of scattering media, Inverse Problems, vol. 18, Institute of Physics Publishing, 2002, pp. 1761-1773.

[11] E. Kerbrat, "Imagerie par retournement temporel en contrôle non destructif des matériaux", PhD Thesis, University of Paris 7, 2001

[12] E. Kerbrat, D. Clorennec, C. Prada, D. Royer, D. Cassereau, M. Fink, Detection of cracks in a thin air-filled hollow cylinder by application of the DORT method to elastic components of the echo, Ultrasonics 40 (2002) 715-720.

[13] D. Clorennec, D. Royer, H. Walaszek, Nondestructive evaluation of cylindrical parts using laser ultrasonics, Ultrasonics 40 (2002) 783-789.

[14] F. Wu, J.L. Thomas, M. Fink, Time reversal of ultrasonics fields - Part 2: experimental results, Ferroelectrics and Frequency Control 39 (5) (September 1992). 
[15] M. Fink, G. Montaldo, M. Tanter, Time reversal acoustics in biomedical engineering, Annual Review of Biomedical Engineering 5 (2003) 465-497.

[16] J. Bercoff, S. Chaffal, M. Tanter, L. Sandrin, S. Catheline, J.L. Gennisson, M. Fink, M. Meunier, In vivo breast tumor detection using transient elastography, Ultrasound Medicine and Biology 48 (2003) 2577-2589.

[17] S. Granger, A. Loukili, G. Pijaudier-Cabot, G. Chanvillard, Experimental characterization of the self-healing of cracks in an ultra high performance cementitious material: mechanical tests and acoustic emission analysis, Cement and Concrete Research 37 (4) (2007) 519-527.
[18] A. Loukili, P. Richard, J. Lamirault, A study on delayed deformations of an ultra high strength cementious material, ACI Recent Advances in Concrete Technology 179 (1998) 929-949.

[19] A. Neville, Autogenous healing - a concrete miracle? Concrete International (november 2002) 76-82.

[20] N. Hearn, C.T. Morley, Self healing property of concrete - experimental evidence Materials and Structures 30 (1997) 404-411.

[21] C. Edvardsen, Water permeability and autogenous healing of cracks in concrete ACI Materials Journal 96 (4) (1999) 448-454. 